\title{
Relationships Among Conventional Cardiovascular Risk Factors and Lifestyle Habits With Arterial Stiffness in Type 2 Diabetic Patients
}

\author{
Misako Hamamura ${ }^{\mathrm{a}}$, Tomoya Mita ${ }^{\mathrm{a}, \mathrm{b}, \mathrm{g}}$, Yusuke Osonoi ${ }^{\mathrm{a}}$, Takeshi Osonoic, Miyoko Saitoc \\ Atsuko Tamasawa ${ }^{c}$, Shiho Nakayama ${ }^{a}$, Yuki Someya ${ }^{a}$, Hidenori Ishida ${ }^{c}$, \\ Masahiko Gosho $^{\text {, }}$, Akio Kanazawa ${ }^{\text {a, e }}$, Hirotaka Watada, b, e, f
}

\begin{abstract}
Background: While conventional cardiovascular risk factors and certain lifestyle habits are associated with arterial stiffness in patients with type 2 diabetes mellitus (T2DM), it is still unknown whether they are actually associated with arterial stiffness even after adjustment for conventional cardiovascular risk factors and lifestyle habits. The aim of this study was to identify variables that are associated with brachial-ankle pulse wave velocity (baPWV).
\end{abstract}

Methods: The study participants comprised 724 Japanese T2DM outpatients free of history of cardiovascular diseases. Lifestyle habits were analyzed using self-reported questionnaires. The associations among conventional cardiovascular risk factors and lifestyle habits with baPWV were investigated by multivariable linear regression analysis.

Results: The mean age of the study subjects was $57.8 \pm 8.6$ years, and $62.8 \%$ of those were males. The mean $\mathrm{HbAlc}$ was $7.0 \pm 1.0 \%$, and the estimated duration of T2DM was $9.9 \pm 7.2$ years. Multiple linear regression analysis that included age and gender demonstrated that age and male sex were positively associated with baPWV. In a model adjusted for numerous conventional cardiovascular risk factors and lifestyle habits, age, duration of T2DM, systolic blood pressure, serum uric acid, urinary albumin excretion and poor sleep quality were positively associated with baPWV, while body mass index was nega-

Manuscript accepted for publication February 13, 2017

aDepartment of Metabolism and Endocrinology, Juntendo University Graduate School of Medicine, 2-1-1 Hongo, Bunkyoku, Tokyo 113-8421, Japan

${ }^{\mathrm{b}}$ Center for Molecular Diabetology, Juntendo University Graduate School of Medicine, 2-1-1 Hongo, Bunkyoku, Tokyo 113-8421, Japan

'Naka Memorial Clinic, 745-5, Nakadai, Naka City, Ibaraki 311-0113, Japan ${ }^{\mathrm{d}}$ Department of Clinical Trial and Clinical Epidemiology, Faculty of Medicine, University of Tsukuba, 1-1-1, Tennodai, Tsukuba, Ibaraki 305-8575, Japan ${ }^{e}$ Center for Therapeutic Innovations in Diabetes, Juntendo University Graduate School of Medicine, 2-1-1 Hongo, Bunkyoku, Tokyo 113-8421, Japan fSportology Center, Juntendo University Graduate School of Medicine, 2-1-1 Hongo, Bunkyoku, Tokyo 113-8421, Japan

'Corresponding Author: Tomoya Mita, Department of Metabolism and Endocrinology, Juntendo University Graduate School of Medicine, 2-1-1 Hongo, Bunkyoku, Tokyo 113-8421, Japan. Email: tom-m@juntendo.ac.jp

doi: https://doi.org/10.14740/jocmr2870w tively associated with baPWV.

Conclusions: In Japanese T2DM, in addition to several conventional cardiovascular risk factors, poor sleep quality was associated with baPWV even after adjustment for numerous conventional cardiovascular risk factors and lifestyle habits.

Keywords: Traditional cardiovascular risk factors; Sleep quality; Arterial stiffness; Type 2 diabetes mellitus

\section{Introduction}

Arterial stiffness is increased in patients with type 2 diabetes mellitus (T2DM), which can potentially increase the risk of morbidity and mortality associated with cardiovascular diseases (CVD) [1]. The brachial-ankle pulse wave velocity (baPWV) is a non-invasive technique often used clinically for assessment of arterial wall stiffness and evaluation of the state of atherosclerosis and prediction of CVD in T2DM patients $[2,3]$.

Previous studies demonstrated that conventional risk factors for CVD, such as age, gender, body mass index (BMI), duration of T2DM, glycemic control, dyslipidemia, systolic blood pressure (SBP), estimated glomerular filtration rate (eGFR), uric acid and albuminuria, are associated with increased arterial stiffness in patients with T2DM [1, 4-6]. However, life style-related problems were not fully taken into consideration in these studies, although the risk of CVD in patients with T2DM correlates with numerous lifestyle problems [7, 8]. In fact, we recently demonstrated that poor sleep quality in patients with T2DM correlates with increased arterial stiffness [7]; however, in that study, we did not take conventional cardiovascular risk factors into full consideration. Another study showed that low physical activity was associated with increased arterial stiffness in newly diagnosed patients with T2DM [8]. On the other hand, the association of other lifestyle habits, such as energy intake, morningness-eveningness, sleep duration and depressive state, with baPWV remains largely unknown in patients with T2DM.

The aim of the present study was to identify lifestyle habits that are associated with baPWV in T2DM patients free of apparent CVD, using a model adjusted for numerous conven- 
tional cardiovascular risk factors and lifestyle habits.

\section{Materials and Methods}

\section{Subjects}

The subjects of this cohort study were recruited from three institutions: the Diabetes Outpatient Clinic of Juntendo University (Tokyo, Japan), Naka Memorial Clinic (Naka, Japan), and Secomedic Hospital (Funabashi, Japan) [7, 9, 10]. The inclusion criteria were as follows: 1) male and female T2DM patients, 2) age $\geq 25$ and $<70$ years, and 3 ) signing consent form for participation in the study. The following exclusion criteria were also applied: 1) type 1 or secondary diabetes, 2) presence of severe infection, recent surgery, or severe trauma, 3 ) history of myocardial infarction, angina pectoris, or stroke, 4) chronic renal failure requiring hemodialysis, 5) liver cirrhosis, 6) moderate or severe heart failure (NYHA/New York Heart Association stage III or higher), 7) active malignancy, 8) pregnancy, lactation, or possible pregnancy, and 9) patients judged as ineligible by the clinical investigators.

A total of 1,032 consecutive subjects were screened. Between June 2013 and January 2014, 906 of 1,032 patients who met the above eligibility criteria were enrolled in this study. Among the 906 patients, 736 patients agreed to participate in the study.

\section{Questionnaire survey}

The self-administered questionnaire survey to evaluate sleep quality used in the present study was the Pittsburgh sleep quality index (PSQI) $[9,11]$. Based on the total PSQI score, patients were divided into three groups: the "good sleep quality group" with PSQI score of $\leq 5$, "average sleep quality group" with PSQI 6 - 8, and "poor sleep quality group" with PSQI $\geq 9$ [12]. We also used the morning-evening questionnaire (MEQ) [13] to evaluate morningness and eveningness in individuals. Briefly, 11 questions allowed for choice and scored from 1 to 4. Two questions allowed for choice and scored $0,2,4$ and 6. One question allowed for choice and scored 0,2,3 and 5. The remaining five questions allowed for choice of time scales and scored from 1 to 5 . The sum of all scores was converted into three-point MEQ scales as follows: scores 16 - 52 represented the evening type, scores 53 - 64 represented the neither type, and scores 65 - 86 was considered the morning type, as reported previously [14].

The participating patients also completed the Beck depression inventory (BDI)-II, which is a 21-item questionnaire that assesses hopelessness, irritability, cognition, guilt, fatigue, weight loss, and sexual interest, representing depression-related symptoms [15]. A high BDI score represents depressive state. Dietary habits during the preceding month were also assessed by the self-administered diet history questionnaire (BDHQ). Briefly, the four-page structured BDHQ includes questions on selected foods and is designed to estimate the dietary consumption of 56 food and beverage items [16]. Physical activity level was assessed with the four-question international physical activity questionnaire (IPAQ) [17], and the results were expressed as metabolic equivalent scores (METs $\cdot \mathrm{h} /$ week). In the above questionnaires, full-time employees and shift workers were labeled as workers [9]. The subjects were also divided into non-smokers, former smokers and current smokers [9].

\section{Blood and urine tests}

Blood samples were collected at the Outpatient Clinic after overnight fast and liver and renal function tests, lipid profile and $\mathrm{HbA1c}$ (national glycohemoglobin standardization program) were measured with standard techniques. Urinary albumin excretion (UAE) was measured by the latex agglutination assay using a spot urine sample. The eGFR was calculated by the formula: eGFR $\left(\mathrm{mL} / \mathrm{min} / 1.73 \mathrm{~m}^{2}\right)=194 \times \mathrm{age}^{-0.287} \times \mathrm{se}-$ rum creatinine $e^{-0.1094}(\times 0.739$ for females $)$ [18].

\section{Measurement of baPWV}

BaPWV was measured using an automatic waveform analyzer (BP-203RPE; Colin Medical Technology, Komaki, Japan), as described previously $[7,9,10]$. Briefly, recording was performed with the patients in the supine position after 5-min rest. Occlusion and monitoring cuffs were placed snugly around both areas in the upper and lower extremities. The pressure waveforms were then recorded simultaneously from the brachial arteries by the oscillometric method. All scans were automatically conducted by well-trained investigators who were blinded to the clinical information. Previous studies confirmed the validity and reproducibility of baPWV measurements [19]. The ankle-brachial index was also measured in all participants. A resting ankle-brachial index of $\leq 0.90$ was considered to reflect the presence of peripheral artery disease. The diagnosis of peripheral artery disease based on the ankle-brachial index was further confirmed by computed tomographic angiography, magnetic resonance angiography or catheter angiography. Based on the data obtained from the above procedures, six patients with arterial lumen narrowing of $\geq 50 \%$ were excluded from analysis.

\section{Statistical analysis}

Results were presented as mean $\pm \mathrm{SD}$ or median (interquartile range: $25-75 \%$ ) for continuous variables or number (proportion) of patients for categorical variables. Several parameters were logarithmically transformed to approximate normal distribution. Multiple linear regression analysis was performed to investigate whether possible cardiovascular risk factors evaluated by clinical, biochemical, metabolic tests and lifestyle habits were associated with baPWV in patients with T2DM free of apparent CVD. Conventional atherosclerotic risk factors based on clinical judgment and certain life styles were included in the model. Statistical tests were two-sided with 5\% significant level. All analyses were performed using the SAS software 
Table 1. Patients' Characteristics $(n=724)$

\begin{tabular}{|c|c|}
\hline Age (years) & $57.8 \pm 8.6$ \\
\hline Gender (male) & $455(62.8)$ \\
\hline Body mass index $\left(\mathrm{kg} / \mathrm{m}^{2}\right)$ & $24.6 \pm 4.1$ \\
\hline Estimated history of diabetes (years) & $9.9 \pm 7.2$ \\
\hline $\mathrm{HbA} 1 \mathrm{c}(\%)$ & $7.0 \pm 1.0$ \\
\hline Systolic blood pressure (mm Hg) & $127 \pm 14$ \\
\hline Diastolic blood pressure (mm Hg) & $77 \pm 11$ \\
\hline Total cholesterol (mg/dL) & $185 \pm 28$ \\
\hline HDL-cholesterol (mg/dL) & $59 \pm 14$ \\
\hline Triglyceride (mg/dL) & $100(70-152)$ \\
\hline AST (U/L) & $21(18-27)$ \\
\hline $\operatorname{ALT}(\mathrm{U} / \mathrm{L})$ & $22(16-33)$ \\
\hline$\gamma-\mathrm{GTP}(\mathrm{U} / \mathrm{L})$ & $25(17-39)$ \\
\hline Uric acid (mg/dL) & $5.5 \pm 1.2$ \\
\hline eGFR $\left(\mathrm{mL} / \mathrm{min} / 1.73 \mathrm{~m}^{2}\right)$ & $78 \pm 18$ \\
\hline UAE (mg/g creatinine) & $10(6-23)$ \\
\hline Current smoker (yes) & $173(23.9)$ \\
\hline Alcohol (g/day) & $12.3 \pm 21.5$ \\
\hline Diabetic retinopathy & $264(36.6)$ \\
\hline \multicolumn{2}{|l|}{ On treatment for $(\mathrm{n}, \%)$} \\
\hline Insulin therapy & $79(10.4)$ \\
\hline Hypertension & $346(47.8)$ \\
\hline Dyslipidemia & $441(60.9)$ \\
\hline \multicolumn{2}{|l|}{ Antiplatelet } \\
\hline Morningness-eveningness questionnaire & $57.4 \pm 7.3$ \\
\hline Pittsburgh sleep quality index & $5.1 \pm 3.0$ \\
\hline Beck depression inventory-II & $9.9 \pm 7.7$ \\
\hline Energy intake (kcal/day) & $1,713 \pm 582$ \\
\hline Physical activity (METs·h/week) & $42.9 \pm 70.5$ \\
\hline Sleep duration $(\mathrm{h})$ & $6.5 \pm 1.2$ \\
\hline baPWV $(\mathrm{cm} / \mathrm{s})$ & $1,543 \pm 280$ \\
\hline
\end{tabular}

Data are presented as mean $\pm \mathrm{SD}$ or number (proportion). ALT: alanine aminotransferase; AST: aspartate aminotransferase; baPWV: brachialankle pulse wave velocity; eGFR: estimated glomerular filtration rate; HDL-C: high-density lipoprotein-cholesterol; UAE: urinary albumin excretion; -GTP: -glutamyl transpeptidase.

version 9.3 (SAS Institute, Cary, NC).

\section{Results}

Among the 736 participating patients, 12 did not complete the questionnaires and/or baPWV measurement and/or had an ankle-brachial index of $\leq 0.90$, and accordingly were excluded from analysis. Table 1 summarizes the characteristics of the remaining 724 Japanese patients with T2DM. The mean age was $57.8 \pm 8.6$ years, and $62.8 \%$ of the subjects were males.
The mean $\mathrm{HbA} 1 \mathrm{c}$ was $7.0 \pm 1.0 \%$, and the estimated duration of T2DM was $9.9 \pm 7.2$ years. Most subjects had attended educational programs in the past about diet and exercise therapy and received appropriate medical treatments.

The results of multivariable linear regression analysis are shown in Table 2. The regression analysis that included age and gender, which are major confounding factors for atherosclerosis, demonstrated that age and male sex were positively associated with baPWV (model 1). In model 2 adjusted for conventional cardiovascular risk factors (+model 1), age, duration of T2DM, $\mathrm{HbA1c}$, SBP, uric acid and UAE were positively associated with baPWV while BMI was negatively associated with baPWV. Almost similar findings were observed in model 3 adjusted for alcohol consumption, smoking, and background therapies for CVD risk factors $(+$ model 2$)$. In model 4 adjusted for lifestyle habits (+model 3), age, duration of T2DM, SBP, uric acid, UAE and poor sleep quality were positively associated with baPWV while BMI was negatively associated with baPWV.

\section{Discussion}

In agreement with previous studies [1, 4-6, 20], our data demonstrated that conventional cardiovascular risk factors, such as age, duration of T2DM, SBP, uric acid and UAE, were associated with increased arterial stiffness in patients with T2DM even after adjustment for some lifestyle habits. Furthermore, we found that poor sleep quality was associated with arterial stiffness even after adjustment for several conventional cardiovascular risk factors and other lifestyle habits. In this regard, a previous report demonstrated that high levels of catecholamines caused by poor sleep quality may be associated with progression of arterial stiffness in healthy middle-aged adults [21]. High catecholamine levels could promote smooth muscle cell proliferation and fibrosis, leading to structural changes in the arterial wall [22]. Although the study design does not allow evaluation of causal relations, the above data suggest that poor sleep quality may be an important target of interventions that can prevent arterial stiffness in patients with T2DM. In contrast, our data failed to show close association of other lifestyle problems, such as energy intake, physical activity, morningness-eveningness, sleep duration and depressive state, with arterial stiffness in patients with T2DM. A recent clinical trial demonstrated that life style interventions, with a special focus on reduced calorie intake and increased physical activity, did not affect the rate of CVD in obese T2DM patients [23]. It is possible that not only caloric intake, but rather dietary composition, may need to be modified to prevent or delay the development of CVD in patients with T2DM.

While previous reports demonstrated that BMI was positively associated with arterial stiffness [5, 20, 24, 25], BMI was inversely associated with arterial stiffness in this study, consistent with a previous report in non-T2DM patients [26]. The exact explanation for the conflicting results is unknown; however, a previous study showed higher baPWV levels in T2DM patients with normal BMI and increased visceral adiposity compared to those with higher BMI and normal visceral adiposity [27]. Generally, increased visceral adiposity is associated with 
Table 2. Results of Multiple Linear Regression Analysis for the Variables Associated With baPWV

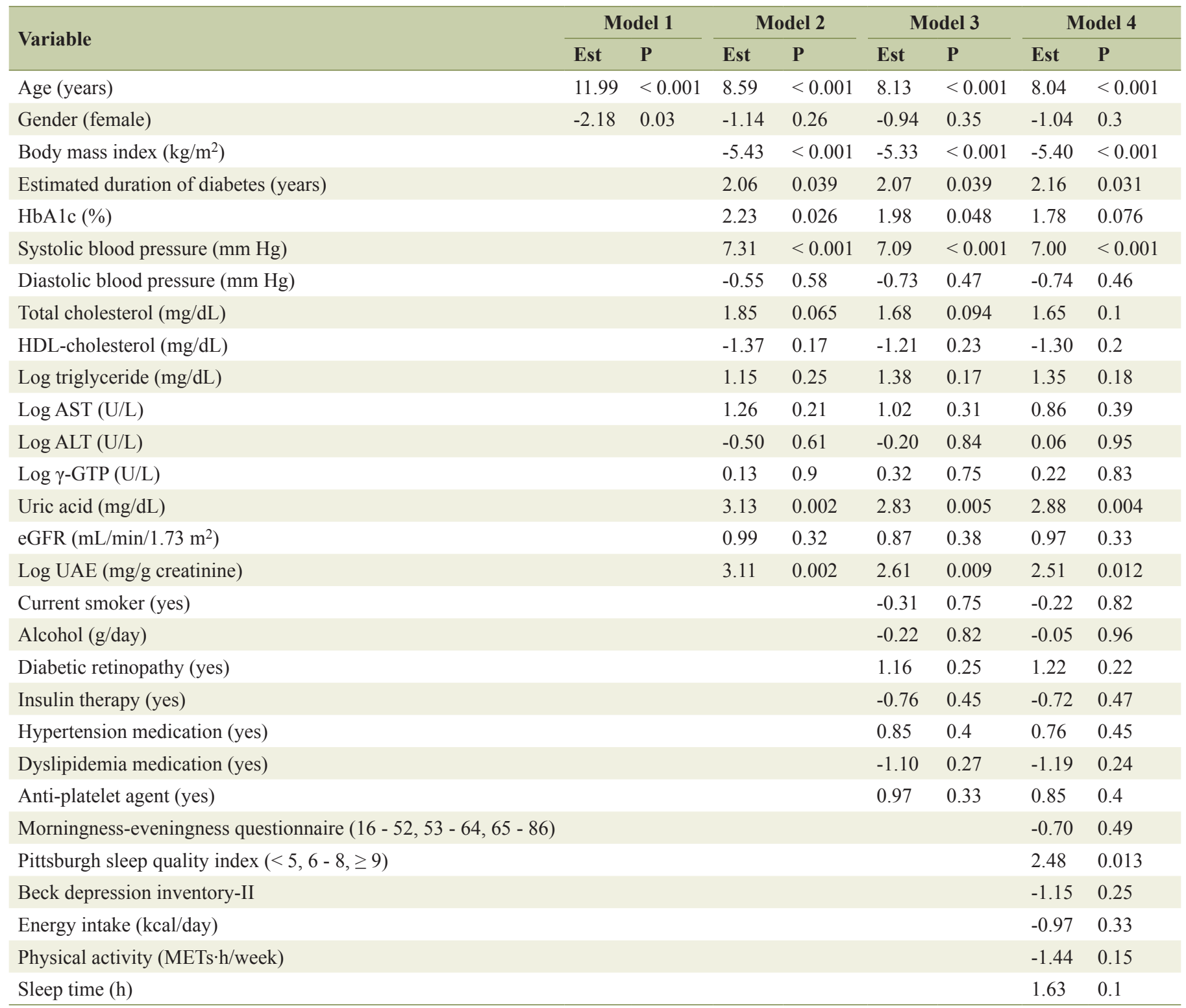

Multiple linear regression analysis included age and gender (model 1), model 1 plus body mass index, estimated duration of diabetes, HbA1c, systolic blood pressure, diastolic blood pressure, total cholesterol, high density lipoprotein-cholesterol, triglyceride, AST, ALT, Y-GTP, serum uric acid, eGFR and UAE (model 2), model 2 plus current smoker, alcohol, diabetic retinopathy, insulin therapy, hypertension medication, hyperlipidemia medication and anti-platelet agents (model 3), model 3 plus morningness-eveningness questionnaire, Pittsburgh sleep quality index, Beck depression inventoryII, energy intake (kcal/day), physical activity (kcal/day) and sleep time (model 4). Est: standardized estimate of regression parameter; ALT: alanine aminotransferase; AST: aspartate aminotransferase; baPWV: brachial-ankle pulse wave velocity; eGFR: estimated glomerular filtration rate; HDL-C: high-density lipoprotein-cholesterol; UAE: urinary albumin excretion; Y-GTP: $\mathrm{Y}$-glutamyl transpeptidase.

insulin resistance, which increases the production of inflammatory cytokines, leading to arterial stiffness. Since Asians tend to have insulin resistance even with normal BMI [28], evaluation of visceral adiposity may be helpful in the assessment of effect of obesity on arterial stiffness in these subjects.

A close association between serum uric acid and arterial stiffness in male patients with newly diagnosed T2DM was reported previously, even after adjustment for several risk factors for atherosclerosis [29]. In the present study, we demonstrated a significant association between serum uric acid and arterial stiffness in a wider range of T2DM population than the above study, even after adjustment for lifestyle habits in addition to numerous cardiovascular risk factors. However, whether serum uric acid plays an important role or is a potentially useful sensitive marker for identifying patients at high risk for CVD is not clear in this cross-sectional study. Indeed, the deleterious effect of hyperuricemia on arterial stiffness may be mediated through other interactive cardiovascular risk factors and/or the 
effect of hyperuricemia may depend on the stage of atherosclerosis $[6,29]$.

The present study has certain limitations. First, the crosssectional design does not allow evaluation of the causal relationship between risk factors for atherosclerosis and arterial stiffness. Second, we only used evaluation of arterial stiffness to assess atherosclerosis. It is important to validate the present findings using other techniques. Third, we cannot rule out the possible effects of other lifestyle habits that we did not assessed in this study on arterial stiffness.

\section{Conclusion}

In conclusion, we found that several conventional cardiovascular risk factors, such as age, duration of T2DM, SBP, serum uric acid, UAE, lower BMI and poor sleep quality were associated with baPWV even after adjustment for multiple traditional cardiovascular risk factors and lifestyle habits.

\section{Acknowledgments}

The authors thank the following staff who participated in this trial: Ms. Risa Yamamoto and Ms. Emi Ito (Department of Metabolism and Endocrinology, Juntendo University Graduate School of Medicine), Ms. Satako Douguchi, Ms. Chiyoko Sato, Ms. Yoko Ono, Mr. Tadanori Koibuchi, Ms. Norie Shiina, Ms. Nana Shiozawa, Ms. Mariko Kobori, Ms. Misato Ojima, Ms. Akiko Haginoya, Ms. Kumiko Fujisaku and Mr. Katuhiro Kawauchi (Naka Memorial Clinic) for the excellent technical support.

\section{Declarations}

The study was approved by the Institutional Review Board of our hospital and conducted in accordance with the principles described in the Declaration of Helsinki. All patients provided written informed consent before participation and registered on the University Hospital Medical Information Network Clinical Trials Registry (UMIN000010932).

\section{Competing Interests}

HM is an employee of Takeda Pharmaceutical Co. HW has received lecture fees from Novo Nordisk, Inc., Eli Lilly and Company, Sanofi, Dainippon Sumitomo Pharma Co., Fujifilm, Bayer Health Care, Kissei Pharmaceutical Company, Mochida Pharmaceutical Company, MSD, Takeda Pharmaceutical Company, Boehringer Ingelheim Pharmaceuticals, Inc., Daiichi-Sankyo, Ono Pharmaceutical Co., Ltd, Novartis Pharmaceuticals Corporation, Mitsubishi Tanabe Pharma Corporation, AstraZeneca LP, Kyowa Hakko Kirin Company, Ltd, Sanwa Kagaku Kenkyusyo Company, Ltd, Kowa Company Ltd, Astellas Pharma, Inc., advisory fees from Novo Nordisk, Inc., Mochida Pharma Company, AstraZeneca LP, Kowa Company, Astellas Pharma, Inc., Sanofi, Boehringer Ingelheim Pharma- ceuticals, Inc., MSD, Mitsubishi Tanabe Pharma Corporation, Novartis Pharmaceuticals Corporation, Dainippon Sumitomo Pharma Co., Takeda Pharmaceutical Company, Ono Pharmaceutical Co., Pfizer, Inc., Kowa Company and research funds from Boehringer Ingelheim, Pfizer, Mochida Pharmaceutical Co., Sanofi-Aventis, Novo Nordisk Pharma, Novartis Pharmaceuticals, Sanwakagaku Kenkyusho, Terumo Corp. Eli Lilly, Mitsubishi Tanabe Pharma, Daiichi Sankyo Inc., Takeda Pharmaceutical Co., MSD, Shionogi, Pharma, Dainippon Sumitomo Pharma, Kissei Pharma, and Astrazeneca.

\section{Author Contributions}

All authors contributed to the study design and were involved at all stages of manuscript development. $\mathrm{MH}$ and TM drafted the manuscript. $\mathrm{MG}$, a statistician, contributed to the analysis of research data. All authors were also involved in the collection, analysis and interpretation of data, reviewed/edited the manuscript and approved the final manuscript. HW is the principal guarantors of this work and has full access to all study data and takes responsibility for the integrity of the data and accuracy of data analysis. All authors have read and agreed to the publication of the manuscript.

\section{Grant Support}

This study was conducted through a research grant from the Manpei Suzuki Diabetes Foundation (to TM). The funding agency had no role in the study design, data collection or analysis, decision to publish, or preparation of the manuscript.

\section{Abbreviations}

ALT: alanine aminotransferase; baPWV: brachial-ankle pulse wave velocity; BDHQ: brief, self-administered diet history questionnaire; BDI: Beck depression inventory; BMI: body mass index; SBP: systolic blood pressure; CVD: cardiovascular disease; eGFR: estimated glomerular filtration rate; $\gamma$-GTP: $\gamma$-glutamyl transpeptidase; HDL-C: high-density lipoproteincholesterol; IPAQ: international physical activity questionnaire; MEQ: morningness-eveningness questionnaire; PSQI: Pittsburgh sleep quality index; T2DM: type 2 diabetes mellitus; UAE: urinary albumin excretion

\section{References}

1. Taniwaki H, Kawagishi T, Emoto M, Shoji T, Kanda H, Maekawa K, Nishizawa Y, et al. Correlation between the intima-media thickness of the carotid artery and aortic pulse-wave velocity in patients with type 2 diabetes. Vessel wall properties in type 2 diabetes. Diabetes Care. 1999;22(11):1851-1857.

2. Nakamura M, Yamashita T, Yajima J, Oikawa Y, Sagara K, Koike A, Kirigaya H, et al. Brachial-ankle pulse wave 
velocity as a risk stratification index for the short-term prognosis of type 2 diabetic patients with coronary artery disease. Hypertens Res. 2010;33(10):1018-1024.

3. Maeda Y, Inoguchi T, Etoh E, Kodama Y, Sasaki S, Sonoda N, Nawata H, et al. Brachial-ankle pulse wave velocity predicts all-cause mortality and cardiovascular events in patients with diabetes: the Kyushu Prevention Study of Atherosclerosis. Diabetes Care. 2014;37(8):2383-2390.

4. Ferreira MT, Leite NC, Cardoso CR, Salles GF. Correlates of aortic stiffness progression in patients with type 2 diabetes: importance of glycemic control: the Rio de Janeiro type 2 diabetes cohort study. Diabetes Care. 2015;38(5):897-904.

5. Liu JJ, Sum CF, Tavintharan S, Yeoh LY, Ng XW, Moh $\mathrm{AM}$, Lee $\mathrm{S}$, et al. Obesity is a determinant of arterial stiffness independent of traditional risk factors in Asians with young-onset type 2 diabetes. Atherosclerosis. 2014;236(2):286-291.

6. Fukui M, Tanaka M, Shiraishi E, Harusato I, Hosoda H, Asano M, Kadono M, et al. Serum uric acid is associated with microalbuminuria and subclinical atherosclerosis in men with type 2 diabetes mellitus. Metabolism. 2008;57(5):625-629.

7. Osonoi Y, Mita T, Osonoi T, Saito M, Tamasawa A, Nakayama S, Someya Y, et al. Poor sleep quality is associated with increased arterial stiffness in Japanese patients with type 2 diabetes mellitus. BMC Endocr Disord. 2015;15:29.

8. Funck KL, Laugesen E, Hoyem P, Fleischer J, Cichosz SL, Christiansen JS, Hansen TK, et al. Low Physical Activity Is Associated With Increased Arterial Stiffness in Patients Recently Diagnosed With Type 2 Diabetes. Am J Hypertens. 2016;29(7):882-888.

9. Osonoi Y, Mita T, Osonoi T, Saito M, Tamasawa A, Nakayama S, Someya Y, et al. Morningness-eveningness questionnaire score and metabolic parameters in patients with type 2 diabetes mellitus. Chronobiol Int. 2014;31(9):1017-1023.

10. Osonoi Y, Mita T, Osonoi T, Saito M, Tamasawa A, Nakayama S, Someya Y, et al. Relationship between dietary patterns and risk factors for cardiovascular disease in patients with type 2 diabetes mellitus: a cross-sectional study. Nutr J. 2016;15:15.

11. Buysse DJ, Reynolds CF, 3rd, Monk TH, Berman SR, Kupfer DJ. The Pittsburgh Sleep Quality Index: a new instrument for psychiatric practice and research. Psychiatry Res. 1989;28(2):193-213.

12. Tsai YW, Kann NH, Tung TH, Chao YJ, Lin CJ, Chang $\mathrm{KC}$, Chang SS, et al. Impact of subjective sleep quality on glycemic control in type 2 diabetes mellitus. Fam Pract. 2012;29(1):30-35.

13. Horne JA, Ostberg O. A self-assessment questionnaire to determine morningness-eveningness in human circadian rhythms. Int J Chronobiol. 1976;4(2):97-110.

14. Taillard J, Philip P, Chastang JF, Bioulac B. Validation of Horne and Ostberg morningness-eveningness questionnaire in a middle-aged population of French workers. J Biol Rhythms. 2004;19(1):76-86.

15. Beck AT, Steer RA, Brown GK. Manual for the Beck De- pression Inventory-II. San Antonio, TX: Psychological Corporation. 1996.

16. Agency SaT. Standard Tables of Food Composition in Japan (in Japanese), 5th rev ed. Tokyo: Printing Bureau of the Ministry of Finance. 2005.

17. Craig CL, Marshall AL, Sjostrom M, Bauman AE, Booth ML, Ainsworth BE, Pratt M, et al. International physical activity questionnaire: 12-country reliability and validity. Med Sci Sports Exerc. 2003;35(8):1381-1395.

18. Matsuo S, Imai E, Horio M, Yasuda Y, Tomita K, Nitta $\mathrm{K}$, Yamagata $\mathrm{K}$, et al. Revised equations for estimated GFR from serum creatinine in Japan. Am J Kidney Dis. 2009;53(6):982-992.

19. Yamashina A, Tomiyama H, Takeda K, Tsuda H, Arai T, Hirose K, Koji Y, et al. Validity, reproducibility, and clinical significance of noninvasive brachial-ankle pulse wave velocity measurement. Hypertens Res. 2002;25(3):359364.

20. Mohammedi K, Compaore A, Potier L, Belhatem N, Feron M, Matallah N, Travert F, et al. Outpatient measurement of arterial stiffness in patients with type 2 diabetes and obesity. J Diabetes. 2017;9(3):237-242.

21. Zhang J, Ma RC, Kong AP, So WY, Li AM, Lam SP, Li SX, et al. Relationship of sleep quantity and quality with 24-hour urinary catecholamines and salivary awakening cortisol in healthy middle-aged adults. Sleep. 2011;34(2):225-233.

22. Zhang H, Faber JE. Trophic effect of norepinephrine on arterial intima-media and adventitia is augmented by injury and mediated by different alpha1-adrenoceptor subtypes. Circ Res. 2001;89(9):815-822.

23. Wing RR, Bolin P, Brancati FL, Bray GA, Clark JM, Coday M, Crow RS, et al. Cardiovascular effects of intensive lifestyle intervention in type 2 diabetes. $\mathrm{N}$ Engl $\mathbf{J}$ Med. 2013;369(2):145-154.

24. Yeboah K, Antwi DA, Gyan B. Arterial Stiffness in Nonhypertensive Type 2 Diabetes Patients in Ghana. Int J Endocrinol. 2016;2016:6107572.

25. Yeboah K, Antwi DA, Gyan B, Govoni V, Mills CE, Cruickshank JK, Amoah AG. Arterial stiffness in hypertensive and type 2 diabetes patients in Ghana: comparison of the cardio-ankle vascular index and central aortic techniques. BMC Endocr Disord. 2016;16(1):53.

26. Yang F, Wang G, Wang Z, Sun M, Cao M, Zhu Z, Fu Q, et al. Visceral adiposity index may be a surrogate marker for the assessment of the effects of obesity on arterial stiffness. PLoS One. 2014;9(8):e104365.

27. Bouchi R, Minami I, Ohara N, Nakano Y, Nishitani R, Murakami M, Takeuchi T, et al. Impact of increased visceral adiposity with normal weight on the progression of arterial stiffness in Japanese patients with type 2 diabetes. BMJ Open Diabetes Res Care. 2015;3(1):e000081.

28. Chan JC, Malik V, Jia W, Kadowaki T, Yajnik CS, Yoon KH, Hu FB. Diabetes in Asia: epidemiology, risk factors, and pathophysiology. JAMA. 2009;301(20):2129-2140.

29. Zhang J, Xiang G, Xiang L, Sun H. Serum uric acid is associated with arterial stiffness in men with newly diagnosed type 2 diabetes mellitus. J Endocrinol Invest. 2014;37(5):441-447. 